\title{
Spontaneous Regression of a Large Symptomatic Calcified Central Thoracic Disc Herniation: A Case Report with a Review of the Literature
}

\author{
Nanjundappa S Harshavardhana ${ }^{1 *}$, Terry P Panvica ${ }^{2}$ and Manuel R Pinto ${ }^{2}$ \\ ${ }^{1}$ Forth Valley Royal Hospital, UK \\ ${ }^{2}$ Twin Cities Spine Center, USA
}

Submission: September 11, 2017; Published: October 04, 2017

*Corresponding author: Dr. Manuel R Pinto, MD, Twin Cities Spine Center, Piper Building, 913 E 26th Street, Suite 600Minneapolis-55404, United States of America, Tel: 16127756200; Email: mrpinto@me.com

\begin{abstract}
Disc herniations in the thoracic spine are rare and the incidence of symptomatic thoracic disc herniation (TDH) is $1 \mathrm{in}$ a million. The incidence of asymptomatic TDH varies from $11-13 \%$. They are classified as small $(\leq 10 \%)$, medium (11-20\%), large (21-40\%), or giant $(\geq 41 \%)$ based on the extent of canal compromise on advanced imaging. The large symptomatic calcified central TDHs are best treated operatively, as the disc fragment could be adherent to the dura or remain intra-dural [1]. We hereby report a case of one such large central calcified TDH at the T7-T8 level in a 46 year old gentleman who presented with axial mid-thoracic back pain and right-sided radicular symptoms along the intercostal nerve distribution, in addition to tingling and numbness in his lower extremities. The calcified extruded intra-dural disc fragment regressed spontaneously over two and half months, while the patient was waiting for a scheduled elective decompression surgery. The relevant MRI and CT scan images with a review of the salient literature are discussed. We believe this to be the first such case of a spontaneous regression of a large symptomatic central calcified TDH to be reported in English literature that is documented with CT and MRI scans.
\end{abstract}

Keywords: Disc herniation; Calcification; Thoracic spine; Spontaneous regression; Radiculopathy; Myelopathy

\section{Introduction}

The thoracic spine is the least common region to present with a disc herniation in comparison to cervical and lumbar regions. Majority of thoracic disc herniations (THDs) are asymptomatic and detected incidentally on magnetic resonance imaging (MRI). The incidence of asymptomatic TDH varies from $11-13 \%$ [2]. They are classified as small $(\leq 10 \%)$, medium $(11-20 \%)$, large $(21-40 \%)$, or giant $(\geq 41 \%)$ based on the extent of canal compromise on advanced imaging studies (i.e. CT and MRI) [1,3]. Thoracic disc herniations (TDHs) are rare and most commonly affect the thoraco-lumbar junction, with the T11-T12 disc being the most frequently herniated due to the greater spinal mobility and weakness of the posterior longitudinal ligament [4]. Up to $75 \%$ of thoracic disc herniations occur below the T8 vertebra, and herniation of the upper thoracic spine is very rare. A large proportion of TDHs are asymptomatic and little is known regarding the natural history of symptomatic TDHs. They affect older individuals most commonly, and a history of a precipitating event or trauma is often absent in at least half to two-thirds of the affected individuals [5]. The symptoms are secondary to chronic spinal cord compression with the calcification of an extruded fragment suggestive of co-existent degenerative changes. Younger individuals may present with a soft disc herniation and a history of acute trauma or a precipitating event that is responsive to non-operative management. Patients with a TDH may present with axial back pain, radiculopathy, and/or myelopathy with a neuro deficit. Males are more commonly affected than females, and surgery is the treatment of choice for large symptomatic calcified central TDHs [6]. The discectomy of TDHs constitutes $0.15-4 \%$ of all disc surgical procedures and the intra-operative localization of the correct surgical level is challenging [7]. A spontaneous regression of a large symptomatic calcified central $\mathrm{TDH}$ is rare and no case report exists to this day to the best of our knowledge in English literature. We hereby report one such case of a spontaneous resorption of a large symptomatic calcified central T7-T8 TDH in a 46 year old gentleman with relevant MRI and CT image illustrations. A review of the relevant literature with some of the proposed theories explaining such a spontaneous regressive phenomenon is also discussed.

\section{Case Report}

A 46 year old, right hand dominant academician presented with a seven month history of axial mid-thoracic back pain with discomfort and occasional radicular pain around the right hemi-thorax to the senior author's office (MRP). The pain 
was associated muscle spasms on the right side of midline that warranted the intake of non-steroidal anti-inflammatory drugs (i.e. NSAIDs). He had tried a structured physical therapy program and chiropractic treatment for few months, which was marginally helpful in alleviating his symptoms. The pain was aggravated during activities that involved vibration and jarring (especially travelling), and his symptoms were partially relieved with rest and NSAIDs. Increasingly, over the recent three months, he began getting tingling and numbness in his lower extremities that initiated this consultation in our office. His Oswestry disability index (ODI) at the first clinic visit was 32\%. The back pain: radicular hemi-thoracic pain ratio was 70:30 at the time of his first consultation. He denied co-existent red flag signs (i.e. bladder or bowel involvement and foot drop). His past medical history was significant for arthritis, hypertension, and type II diabetes mellitus. His past surgical history included a herniorraphy in his early twenties for an inguinal hernia. He was otherwise in good health and denied any recent history of weight loss, night pains, loss of appetite, fever with night sweats, or chills and rigors. At his most recent annual physical check-up, the assessment by his primary care physician was negative for any concerns of malignancy, hemochromatosis, hyperparathyroidism, gout, pseudogout, and hypercalcemic states. His personal history was negative for the use of tobacco products and his alcohol consumption was minimal $(<3$ drinks per week).

On clinical examination by the senior author (MRP), he had some midline tenderness in the mid-thoracic region over the spinous processes with a good range of motion (ROM) of both the cervical and lumbar spine. Motor strength testing revealed normal strength, tone and power (i.e., Medical Research Council [MRC] grade 5/5 motor strength) in his lower extremities. He had right-sided radiculopathy/anterior-chest band like discomfort with hemi-thoracic tenderness along the course of the T7T9 dermatomal distribution. His right shoulder and scapular function were normal and negative for intrinsic shoulder pathology. The deep tendon reflexes in his knees and ankles were normal. The Romberg's sign was negative, his gait was normal, and he effortlessly performed tandem-walking.

A working diagnosis of a potential space occupying lesion (SOL) in the thoracic region, causing mechanical compression of the spinal cord and/or nerve $\operatorname{root}(\mathrm{s})$, was suspected. An immediate MRI scan was arranged, which revealed a large mass causing the ventral effacement of CSF flow / signal at the T7T8 level. His T2 weighted MRI images are illustrated in (Figure 1a \& 1b). The differential diagnosis included disc herniation and other benign intra-axial neoplasms (i.e. meningioma and schwannoma). A CT scan was requested to further understand the lesion, study the extent of calcification / bony, and the intradural involvement (if any). The key CT sagittal and axial images are depicted in (Figure 2a \& 2b). From these, the diagnosis narrowed down to a large central calcified TDH, following a discussion at the multi-disciplinary team (MDT) meeting.

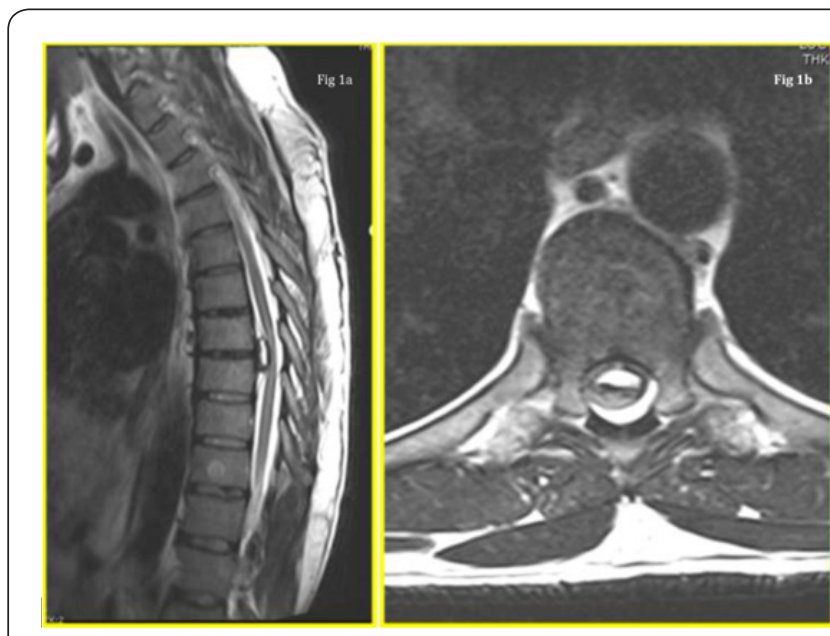

Figure 1a \& 1b: Sagittal and axial T2 images of the thoracic spine depicting the T7-T8 large central thoracic disc herniation (TDH).

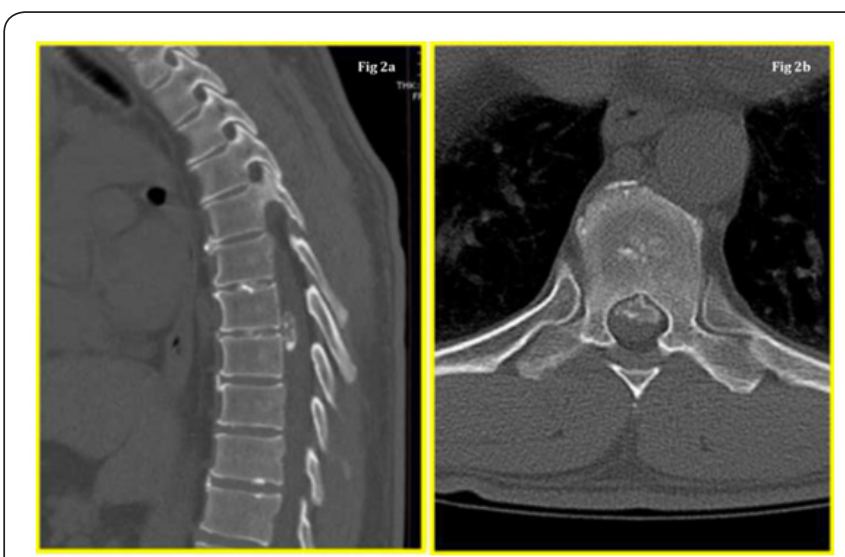

Figure 2a \& 2b: Sagittal and axial CT bone window images of the thoracic spine confirming the calcification of the T7-T8 thoracic disc herniation (TDH).

The patient was seen at the office a week later to discuss the results of his CT and MRI scans. The natural history of disc herniation, available treatment options, risks, complications, prognosis, including watchful waiting, were extensively discussed. Surgical management, using an anterior trans-thoracic approach with a vascular co-surgeon for access, was discussed at length. A surgical discectomy with decompression was recommended and offered, given that he was persistently symptomatic with lower extremity tingling and numbness for at least three months, and because non-operative treatment failed. He chose to electively schedule the surgery in two months (seeking time off work for his post-operative recovery and making alternative arrangements for his academic/professional commitments) with an understanding that it would be undertaken immediately should there be a change in neurology and/or any bladder and bowel involvement.

He was seen in the pre-assessment clinic two weeks prior to the scheduled surgical date, reporting spontaneous improvement in his back pain and anterior-chest band like tightness, and with a resolution of his lower extremity tingling/numbness. His ODI 
was $4 \%$. The proposed surgery was withheld and an interval MRI scan of his thoracic spine was requested. The T2 weighted images at two months from his initial MRI revealed the regression of the large central calcified TDH and this is illustrated in (Figure 3a \& 3b). He had a complete resolution of his symptoms while waiting for surgery and he had normal neurology.He signed a written consent form and permitted us to submit and publish his clinical course of events as a case report provided he remained anonymous.

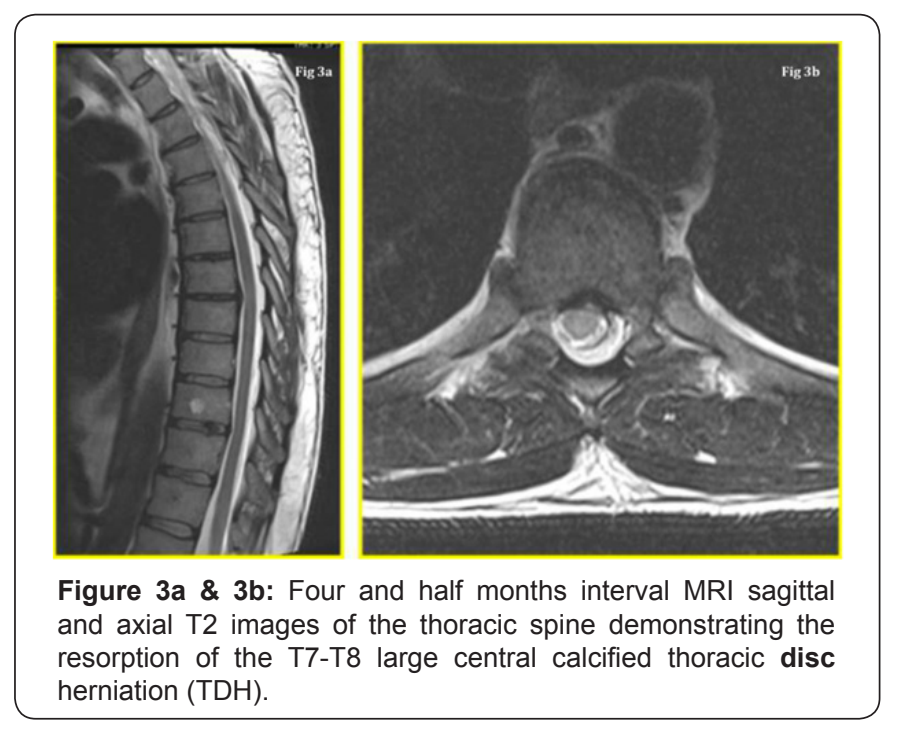

\section{Discussion}

We have reported a case of a large central calcified TDH that has spontaneously regressed over four and half months while the patient was waiting for surgical decompressive surgery. The treatment of choice for a symptomatic large central calcified TDH is operative excision by the anterior trans-throacic approach [8]. Spontaneous regressions of such central calcified discs are very rare and we believe this to be the first such reported case in English literature. The only other case report we could identify in English was a calcified right-sided foraminal T7-T8 TDH with a nucleus pulposus calcification in a 36 year old woman that regressed spontaneously over three months, as reported by Piccirilli et al. [9]. Though interestingly, our case also had the disc herniation occur at the same level (i.e. T7-T8), but it was strikingly different and unique in several aspects, in that our case had

a) A large central calcified TDH,

b) The absence of nucleous pulposus calcification, and

c) A decade older male patient (46 year old gentleman vs. 36 year old woman).

The pathogenesis of this rare phenomenon of spontaneous resorption is poorly understood. Proposed theories postulated to explain it include [10-12]: a) Dehydration of the herniated fragment and its subsequent resorption,

b) Activation of the inflammatory process leading to degradation and resorption,

c) Re-accommodation of the nucleus pulposus in the inter vertebral disc space, or

d) The bathing of extruded disc material in epidural space by blood vessels, which facilitate neovascularisation and eventual macrophage infiltration with phagocytosis.

The clinical course of a TDH is variable, and a patient can present with non-spinal symptoms and an atypical pain pattern mimicking other organ system involvement to frank myelopathy. Though the spinal cord is smallest in diameter in the thoracic region, the cord: canal ratio is at least $40 \%$ compared to $25 \%$ in the cervical region, which make sit sensitive to compressive effects [4]. Varying degrees of myelo-radiculopathy, Brownsequard syndrome to paraplegia are reported with symptomatic TDHs [13]. The incidence of bladder and/or bowel involvement in TDHs varies from $15-20 \%$. A high degree of clinical suspicion, supplemented by a thorough history, physical examination, and confirmation by the appropriate diagnostic imaging, is essential for accurate diagnosis.

Calcification of inter vertebral discs could potentially occur at three places:

a) The annulus fibrosus,

b) The fibro cartilaginous plate, and

c) Centrally in the nucleus pulposus.

Von Lushka was the first to describe this phenomenon in adults and observed such calcifications, especially in the annulus fibrosus, to be almost always asymptomatic [14]. The calcification of the nucleus pulposus is secondary to the deposition of amorphous calcium salts in degenerated tissue and might regress spontaneously, especially in children. Disc calcification also correlated significantly with the morphologic degree of degeneration. CT myelography is considered to be the investigation of choice for the evaluation of calcified TDHs, as it demonstrates the degree of canal encroachment / intra-dural extension better than other imaging modalities [15].

Giant TDHs occupy at least $40 \%$ of the canal dimensions and were first defined by Hott et al. [3]. They produced severe neurological deficit and were associated with poor functional outcome compared to small and medium TDHs. The authors recommended open thoracotomy, rather than thoracoscopy, in treating such giant calcified TDHs. Barbanera et al. [16] reported a series of 7 patients who had symptomatic giant calcified TDHs and none of them regressed spontaneously. Brown et al. [17] evaluated 55 patients with symptomatic TDHs and observed only 15 patients needed surgery (27\%). Encouraged by those findings, they recommended a less aggressive surgical approach, as TDHs 
did not necessarily cause severe neurological compromise. Wood et al. [2] studied the natural history of asymptomatic TDHs and recommended mere observation to be justifiable, even in symptomatic individuals whose chief complaint was axial spinal pain. Outside of English literature, we identified only four cases (three publications) of a spontaneous resorption of a TDH in the French and Italian languages.

Coevoet et al. [18] reported a case of a T9-T10 protrusion that regressed spontaneously over 13 months. Martinez-Quinone et al. [19] reported a case of a T6-T7 TDH in a 47 year old male that regressed spontaneously. Neither of the two reports specified if those discs were calcified. Eap et al. [20] reported two cases of a spontaneous resorption of a calcified TDH. They included a left T8-T9 foraminal herniation in a 48 year old and a large central T12-L1 herniation (similar to our case) in a 45 year oldpatient.

Komori et al. [21] observed extensive rim enhancement on follow-up MRI imaging to be predictive of spontaneous resorption. A gadolinium enhanced diethylenetriaminepentaacetic acid (GdDTPA) MRI could be used to evaluate the neovascularisation zone and might give an insight into the dynamics of disc resorption. Auito et al. [22] studied the determinants of spontaneous resorption in lumbar disc herniation by longitudinal Gd-DTPA enhanced MRI scans, focusing on the rim enhancement zone, and they observed that a higher rim enhancement thickness, a higher degree of HNP displacement, and the age group of 41-50 years to be associated with better resorption rate. The clinical symptom alleviation correlated well with the rate of disc resorption, and dramatic clinical improvement was seen at the end of two months. Unfortunately no such studies have been reported until now for TDHs.

\section{Conclusion}

In summary, a large symptomatic calcified central TDH can be treated with careful observation, as long as the patients are capable of coping with the pain and do not have any lower extremity motor weakness or other red flag signs. A better understanding of pathomechanisms that attempt to further elucidate the complex interplay between disc calcification, disc angiogenesis, and disc degeneration is desired, so that strategies are developed to predict disc herniations that regress spontaneously. Studies focusing on investigating such link mechanisms are desired and constitute grounds for further research. Such studies might also explain which TDHs would most likely regress on their own and define the role of surgery vs. non-operative treatment in the management of symptomatic large central calcified TDHs.

\section{References}

1. Hott JS, Feiz-Erfan I, Kenny K, Dickman CA (2005) Surgical management of giant herniated thoracic discs: analysis of 20 cases. J Neurosurg Spine 3(3): 191-197.

2. Stillerman CB, Weiss MH (1992) Management of thoracic disc disease. Clin Neurosurg 38: 325-352.

3. Wood KB, Blair JM, Aepple DM, Schendel MJ, Garvey TA, et al. (1997) The natural history of asymptomatic thoracic disc herniations. Spine (Phila Pa 1976) 22(5): 525-529.
4. Vanichkachorn JS, Vaccaro AR (2000) Thoracic disk disease: diagnosis and treatment. J Am Acad Orthop Surg 8(3): 159-169.

5. El-Kalliny M, Tew JM, van Loveren H, Dunsker S (1991) Surgical approaches to thoracic disc herniations. Acta Neurochir (Wien) 111(12): 22-32.

6. Vollmer DG, Simmons NE (2000) Transthoracic approaches to thoracic disc herniations. Neurosurg Focus 9(4): 1-6.

7. Yoshihara H (2014) Surgical treatment for thoracic disc herniation: an update. Spine (Phila Pa 1976) 39(6): E406-E412.

8. Mulier S, Debois V (1998) Thoracic disc herniations: transthoracic, lateral, or posterolateral approach? A review. Surg Neurol 49(6): 599606.

9. Piccirilli M, Lapadula G, Caporlingua F, Martini S, Santoro A (2012) Spontaneous regression of a thoracic calcified disc herniation in a young female: a case report and literature review. Clin Neurol Neurosurg 114(6): 779-781.

10. Kato T, Haro H, Komori H, Shinomiya K (2004) Sequential dynamics of inflammatory cytokine, angiogenesis inducing factor and matrix degrading enzymes during spontaneous resorption of the herniated disc. J Orthop Res 22(4): 895-900.

11. Haro H, Kato T, Komori H, Osada M, Shinomiya K (2002) Vascular endothelial growth factor (VEGF)-induced angiogenesis in herniated disc resorption. J Orthop Res 20(3): 409-415.

12. Yasuma T, Arai K, Yamauchi Y (1993) The histology of lumbar intervertebral disc herniation. The significance of small blood vessels in the extruded tissue. Spine (Phila Pa 1976) 18(13): 1761-1765.

13. Kumar A (1991) Thoracic disc prolapse in calcified discs. Orthopedics 14(1): 98-99.

14. Roberts S, Evans H, Trivedi J, Menage J (2006) Histology and pathology of the human intervertebral disc. J Bone Joint Surg Am 88(Suppl 2): $10-14$.

15. Alvarez O, Roque CT, Pampati M (1988) Multilevel thoracic disk herniations: CT and MR studies. J Comput Assist Tomogr 12(4): 649652 .

16. Barbanera A, Serchi E, Fiorenza V, Nina P, Andreoli A (2009) Giant calcified thoracic herniated disc: considerations aiming a proper surgical strategy. J Neurosurg Sci 53(1): 19-25.

17. Brown CW, Deffer PA, Akmakjian J, Donaldson DH, Brugman JL (1992) The natural history of thoracic disc herniation. Spine (Phila Pa 1976) 17(Suppl 6): S97-S102.

18. Coevoet V, Benoudiba F, Lignieres C, Said G, Doyon D (1997) Spontaneous and complete regression in MRI of thoracic disk herniation. J Radiol 78(2): 149-151.

19. Quinones JVM, Escario JA, Consolini F, Calvo RA (2010) Spontaneous regression from intervertebral disc herniation. Propos of a series of 37 cases. Neurocirugia (Astur) 21(2): 108-117.

20. Eap C, Bennis S, Blauwblomme T, Compaore P, Chamsedine A, et al. (2012) Spontaneous resorption of thoracic calcified disc herniation: Report of two cases and review of the literature. Neurochirurgie 58(6): 353-357.

21. Komori H, Okawa A, Haro H, Muneta T, Yamamoto H, et al. (1998) Contrast-enhanced magnetic resonance imaging in conservative management of lumbar disc herniation. Spine (Phila Pa 1976) 23(1): 67-73.

22. Autio RA, Karppinen J, Kurunlahti M, Kyllonen E, Vanharanta H et al. (2002) Gadolinium diethylenetriaminepentaacetic acid enhancement in magnetic resonance imaging in relation to symptoms and signs among sciatic patients: a cross-sectional study. Spine (Phila Pa 1976) 27(13): 1433-1437. 